\title{
Comparison of adipose particle size on autologous fat graft retention in a rodent model
}

\author{
Xiaonan Yang ${ }^{1,2, \#, ~ F r a n c e s c o ~ M . ~ E g r o, " \#, ~ T a r a n e h ~ J o n e s ~}{ }^{3}$, W. Vincent Nerone ${ }^{1}$, Michael Yousefpour $^{1}$, Jeffrey \\ A. Gusenoff', J. Peter Rubin ${ }^{1,4}$, Lauren E. Kokai ${ }^{1,4}$

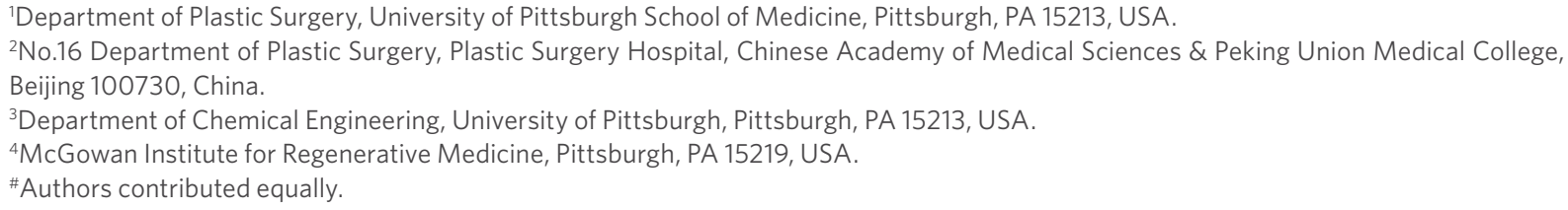

Correspondence to: Dr. Lauren E. Kokai, Department of Plastic Surgery, University of Pittsburgh School of Medicine, Pittsburgh, PA 15213, USA.E-mail: kokail@upmc.edu

\begin{abstract}
How to cite this article: Yang X, Egro FM, Jones T, Nerone WV, Yousefpour M, Gusenoff JA, Rubin JP, Kokai LE. Comparison of adipose particle size on autologous fat graft retention in a rodent model. Plast Aesthet Res 2020;7:8.

http://dx.doi.org/10.20517/2347-9264.2019.63
\end{abstract}

Received: 27 Nov 2019 First Decision: 17 Jan 2020 Revised: 3 Feb 2020 Accepted: 14 Feb 2020 Published: 27 Feb 2020

Science Editor: Jian-Xing Song Copy Editor: Jing-Wen Zhang Production Editor: Tian Zhang

\begin{abstract}
Aim: Unpredictable retention outcomes remain a significant issue in autologous fat grafting procedures. Liposuction cannula variation leads to variability in fat particle size. Recent data suggest that the size of fat particles is closely related to graft healing outcomes; however, this remains a point of contention due to potential confounding variables such as tissue trauma with harvest. The aim of this study was to compare autologous fat grafting outcomes with variable fat particle sizes in an animal model which isolated fat particle size as the primary experimental variable. The overall goal of this work was to determine if reducing fat particle size is an effective method for enhancing graft retention in autologous fat grafting.
\end{abstract}

Methods: The range of fat particle diameter harvested by four common liposuction cannulas was quantified to define relevant small and large particle target diameters. To determine if particle size impacted nutrient and oxygen permeability, small and large particles were incubated in vitro in a spinner flask with an abundance of culture media and vascular endothelial growth factor secretion was measured with enzyme-linked immunosorbent assay. Finally, small and large fat grafts were prepared from subcutaneous mouse fat pads and grafted in syngeneic Balb/CJ mice. Weight and volume retention were evaluated at 1, 4, 8, and 12 weeks. Histological analysis with Masson's trichrome and perilipin 
immunofluorescent staining was performed. Real-time quantitative polymerase chain reaction was performed for adipogenic, inflammatory and apoptotic genes.

Results: The range of fat particle diameters harvested with four commonly used cannulas was 2-7 mm. In vitro studies showed that 5-7-mm particles had significantly increased VEGF secretion normalized to weight, indicating increased tissue hypoxia in these particles compared to 2-4-mm particles. Surprisingly, in vivo comparison in two unique studies showed 2-4-mm and 5-7-mm fat particles had comparable graft retention ( $P=0.5329)$. Masson's trichrome staining revealed increased extracellular matrix and fibrosis in the $5-7-\mathrm{mm}$ particle group $(P=0.0115)$. Adipocyte survival with perilipin demonstrated comparable viability. Gene expression showed large particles experienced increased inflammation and apoptosis at one week after grafting, but overall there were no significant differences between groups.

Conclusion: The ideal fat particle size should be large enough to contain adequate mesenchyme while not so thick as to preclude imbibition. This study suggests that, despite changes in hypoxia and VEGF levels, differing fat particles (2-4-mm and 5-7-mm) can achieve similar graft retention.

Keywords: Fat grafting, particle size, lipofilling, lipoharvesting, adipocyte viability, clinical translation

\section{INTRODUCTION}

Autologous adipose tissue transfer, or fat grafting, is a common procedure in aesthetic and reconstructive plastic surgery with approximately 90,000 patients treated each year in the US alone $e^{[1]}$. Fat grafting allows permanent tissue volume augmentation and correction of contour irregularities in a minimally invasive and safe manner ${ }^{[2]}$. However, studies have demonstrated unpredictable and unsatisfactory graft retention in some patients that can range between $40 \%$ and $90 \%{ }^{\left[{ }^{3}\right]}$. Thus, significant efforts have been undertaken to understand the biology of fat grafting and optimize each step of the fat grafting process from end-to-end (harvesting, processing, and injection) ${ }^{[4-9]}$.

In modern liposuction, excess adipose tissue is extracted through a hollow-bore cannula, typically between 12 and $14 \mathrm{G}$, with variable numbers and sizes of holes. The resulting lipoaspirate product is a suspension of solid adipose tissue particles in liquid comprised of tumescent fluid, blood, and oil from lysed cells. From this lipoaspirate, additional processing steps may be added to remove fluid and oil, ultimately producing a collection of fat particles that are then grafted into the volume void. It is well known in the literature that fat grafts depend on diffusion of oxygen and nutrients from the recipient bed until new blood vessels are formed ${ }^{[10,11]}$. According to work by Khouri et al. ${ }^{[12]}$, when fat particles are very large (greater than $0.16 \mathrm{~cm}$ in radius), oxygen and nutrient diffusion to the interior particle core is insufficient to maintain tissue viability resulting in central necrosis and potentially oil cyst formation. These oil cysts are eventually resorbed through macrophage clearance, resulting in overall graft tissue loss and inferior long-term fat grafting outcomes.

The predominate theory in fat graft preparation is therefore to minimize particle size to as small as possible to reduce central necrosis and ultimately increase graft retention. However, other groups have challenged this assertion, hypothesizing that small cannula diameters increase shear stress in tissue, thereby damaging viability and increasing graft resorption ${ }^{[13,14]}$. In two unique studies, Erdim et al. ${ }^{[15]}$ and Kirkham et al. ${ }^{[16]}$ demonstrated that large diameter cannulas lead to higher graft retention, improved adipocyte survival, and less inflammatory infiltrate and fibrosis. In another comparative study in rabbits, fat was harvested with a regular $4.5-\mathrm{mm}, 1-\mathrm{mL}$ syringe with a sharp steel sleeve, excised , and cut into 1-mg pieces and aspirated with a 2 -mm harvesting cannula, and long-term retention was equal in all groups ${ }^{[17]}$. Therefore, the impact of fat particle size on survival and resorption outcomes remains controversial. 


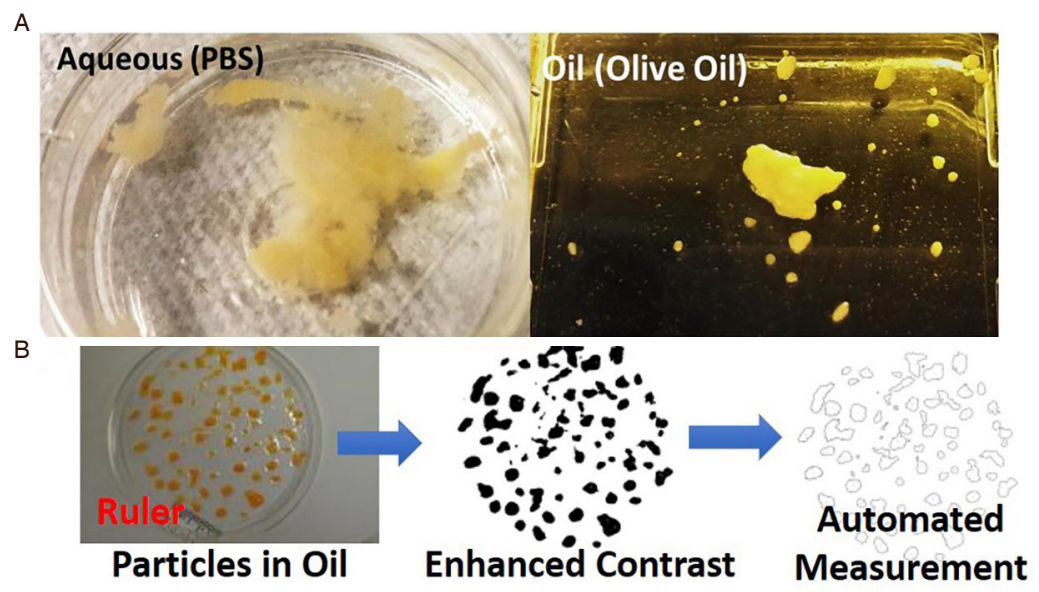

Figure 1. A: comparison of fat particles dispersed in aqueous solution (PBS) or Oil (Olive Oil) showing increased definition of particle boundary in lipophilic solutions; B: adipose particle measurements were obtained by imaging a minimum of 50 particles dispersed in oil with a ruler included in each photograph. Images were then processed with ImageJ to increase particle contrast and the maximum distance between the particle center and the edge was determined. PBS: phosphate-buffered saline

The aim of this study was to compare autologous fat grafting outcomes with variable fat particle sizes in an animal model, which isolated fat particle size as the primary experimental variable. We elected to use a syngeneic mouse model to isolate the variable of fat particle size from confounding factors such human donor tissue variability, trauma from tissue harvest, ischemic time, and recipient tissue bed vascularity. Our overall study goal was to clarify if smaller fat particles result in better fat grafting outcomes in terms of graft retention, histological architecture, adipocyte viability, and neovascularization.

\section{METHODS}

\section{In vitro analysis of human adipose particle size and hypoxia}

Lipoaspirate fat particle size analysis

For the purposes of this study, the range of "typical" fat parcel size was determined by measuring the diameters of adipose particles produced by four commonly used harvesting cannulas for fat grafting procedures: 15-cm Coleman-bucket (two opposing holes) aspiration cannula (Mentor Texas L.P., TX, USA \#COL-ASPI), Khouri 12-hole harvesting cannula [Marina Medical Inc. FL, USA; \#800-205 (12-hole design)], Mercedes tip 3-hole cannula (Grams Medical Inc., Calif., USA), and Shippert cannula (Shippert Medical Co., USA). To obtain lipoaspirate, full thickness skin was obtained as discarded tissue from three different elective body contouring procedures under University of Pittsburgh IRB exemption (PRO13090506). The skin was divided into four even areas with a surgical marker and evenly infused with $0.9 \% \mathrm{NaCl}$ solution. Cannulas were connected to a $20-\mathrm{mL}$ syringe and negative pressure was applied by hand. At least $10 \mathrm{~mL}$ of adipose particles were obtained with each cannula type in unique tissue segments.

To measure the size of fat parcels, lipoaspirate was washed with phosphate buffered saline to remove oil and blood and then tissue particles were dispersed in olive oil and photographed for automated particle analysis [Figure 1A]. Olive oil was selected as the solution of choice after comparing the resolution of particle boundaries in hydrophobic and hydrophilic (PBS) solutions and selecting for the one providing the sharpest particle boundary. Glass calibration beads (QAQC Lab/Coffee Laboratory, White Stone, VA. Cat \#s 600 ZSICSA-2.00, 600 ZSICSA-3.35) of known diameter were used to validate the technique. High resolution images containing at least 50 particles adjacent to a ruler were taken with a Nikon camera. ImageJ was used to enhance image contrast, and particle diameter analysis was performed by measuring the greatest particle diameter [Figure 1B]. 

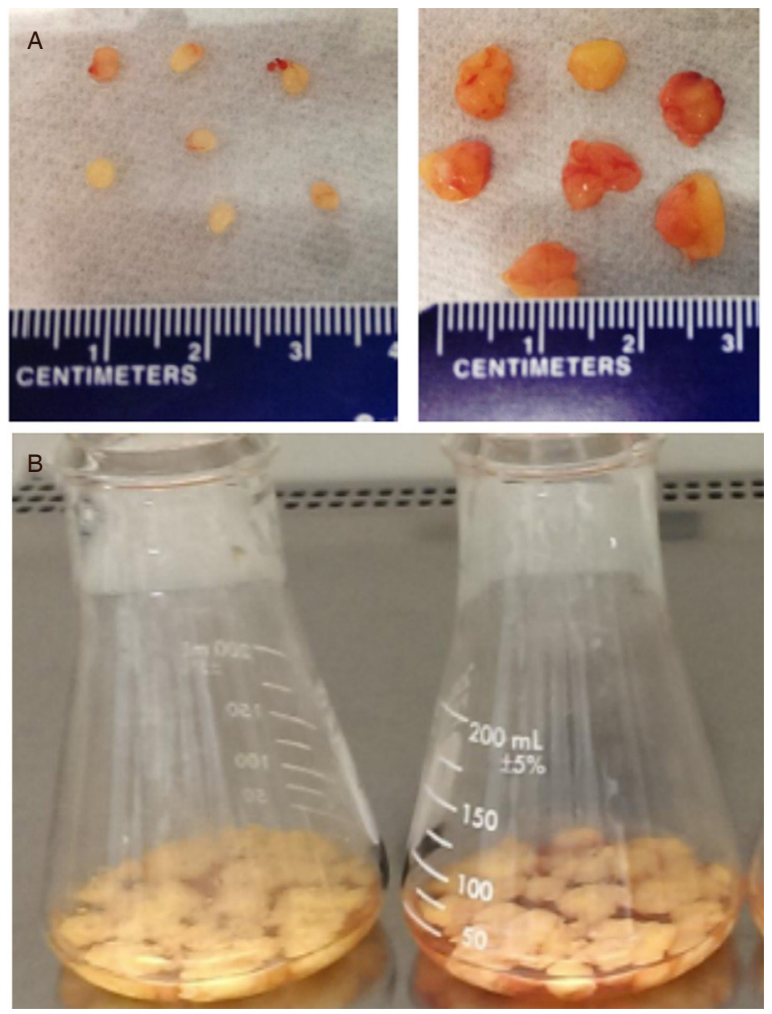

Figure 2. Ex vivo study with adipose particles and VEGF secretion. Small (2-4 mm) and large $(5-7 \mathrm{~mm})$ particles were prepared from whole adipose tissue using surgical scissors and a sterile ruler. Each particle was assessed by weight to ensure consistency. Particles were submerged in sterile cell culture media in a flask and stirred continuously with a stir bar in a humidified CO2 incubator. VEGF: vascular endothelial growth factor

Ex vivo quantification of particle hypoxia

After determining the typical diameter range of fat parcels obtained with four common fat grafting aspiration cannulas, we artificially created particles of the lower end if the size range $(2-4 \mathrm{~mm})$ and upper range (5-7 mm), representing "small" and "large" particles, respectively. To determine if the 5-7-mm particle group incurred oxygen diffusion limitations and increased hypoxia compared to the 2-4-mm group, we cultured particles for seven days in conditions with free access to glucose and oxygen and measured vascular endothelial growth factor secretion ${ }^{[18,19]}$ [Figure $2 \mathrm{~A}$ ]. Full thickness skin samples were obtained from a single donor after body contouring surgery under IRB exemption and placed in a biosafety cabinet for processing. Pieces of adipose tissue for each desired size range were prepared using surgical scissors and subsequently placed into sterile flasks with vented caps with 1:1 (volume) ratio of culture media to fat particles. The total weight of adipose tissue and volume of media in all flasks was equal [Figure 2B]. Particles were constantly stirred inside of a $37^{\circ} \mathrm{C}$ incubator, at $5 \% \mathrm{CO}_{2}$. Half of the media was refreshed every other day up to seven days. Media concentration of vascular endothelial growth factor (VEGF) was measured with enzyme-linked immunosorbent assay (ELISA) according to manufacturer's instructions.

\section{In vivo analysis of fat particle survival in mouse autograft model}

Animals

All animal experiments were performed under approved protocols by the University of Pittsburgh Institutional Animal Care and Use Committee (Protocol\# 12080782). In total, 47 four-week-old female Balb/CJ (The Jackson Laboratory, ME, USA) mice were used: 18 mice for fat grafting and 17 for fat harvesting. All animals were housed under controlled environmental conditions with a 12-h/12-h light/ dark cycle. Standard laboratory chow and sterilized water were provided ad libitum. 


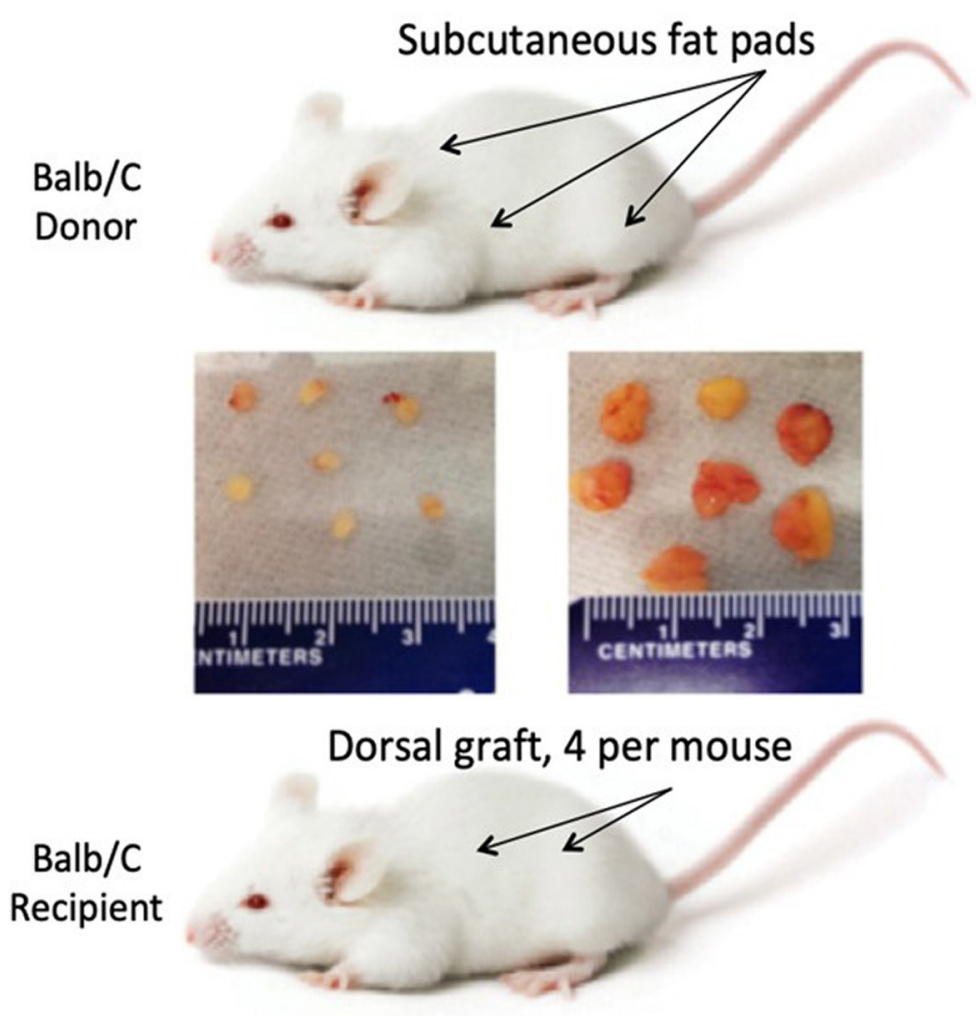

Figure 3. Animal study design schematic. Subcutaneous fat pads were harvested from Balb/C mice. Fat particles with two diameter sizes were prepared with sterile surgical instruments and then immediately syngeneically-transplanted into dorsal subcutaneous spaces via small skin incisions, two points per side in one mouse. At least $n=10$ particles were included in each experimental group at each timepoint

Fat particles preparation and transplantation

Animals were weighed and anesthetized with isoflurane inhalation. The surgical team comprised of two operators, one who harvested fat particles and a second who immediately implanted grafts, minimizing ischemic time and maintaining consistency across the study [Figure 3]. Inguinal and axillary subcutaneous fat pads were harvested in a biosafety cabinet with sterile instruments and immediately weighed with a microelectronic balance (Sartorius, Data Weighing Systems Inc., IL, USA). Simultaneously, the recipient site in a unique, but genetically identical anesthetized mouse was prepared by making a small skin incision on the dorsal flank with scissors and preparing a subcutaneous pocket by opening scissor blades. Immediately after graft preparation, the tissue particles were placed individually into the recipient site and the incision was closed with skin glue (Vetbond, No. NC9604126; Fisher Scientific). A single dose of analgesics, $5 \mathrm{mg} /$ kg ketoprofen (Fort Dodge Animal Health, Overland Park, KS), was administered into the nuchal subcutis.

\section{Evaluation of grafted fat particles}

At defined study timepoints $\left(1,4,8\right.$, and 12 weeks), animals were weighed, euthanized by $\mathrm{CO}_{2}$, and fat particles were explanted, weighed, and measured by gas volume displacement using an AccuPyc II 1340 gas Pycnometer (Micrometrics, Norcross, GA). After measurement, grafts were immediately submerged in 10\% neutral buffered formalin for at least $24 \mathrm{~h}$, processed, and embedded in paraffin for sectioning.

\section{Histological analysis}

Formalin fixed, paraffin embedded samples were sectioned to $6-\mu \mathrm{m}$ sections affixed to charged slides. Masson's trichrome and perilipin immunofluorescent staining were performed on the explants $(n=6)$ at 1-, 4-, 8-, and 12-week timepoints. The sections were observed under a Keyence microscope (Keyence Corp., 

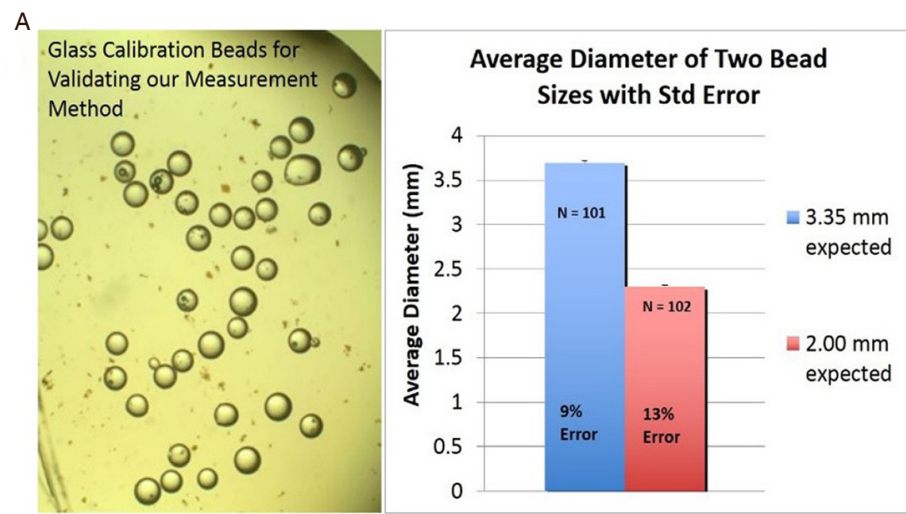

Particle Radius by Harvesting Cannula Brand

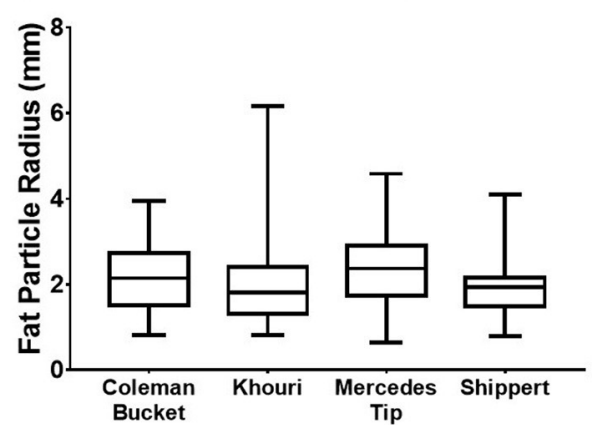

Figure 4. Comparison of fat particle radii from typical harvesting cannulas. Mean $\pm \mathrm{SD}$ of at least 50 particles from three unique donors are presented

IL, USA). The percentage of area of extracellular matrix and fibrosis was evaluated using Image J (National Institutes of Health, Bethesda, MD).

Real-time quantitative polymerase chain reaction

Real-time quantitative polymerase chain reaction (qRT-PCR) was used to compare the relative expression of genes related to adipocyte function [fatty acid binding protein $4(\mathrm{FABP} 4)]^{[20]}$, inflammation [tumor necrosis factor $(\mathrm{TNF} \alpha)$ and interleukin $\left.1(\mathrm{IL} 1)^{[21]}\right]$ and cell stress [glutathione peroxidase $(\mathrm{GPX} 1)^{[22-24]}$ and Caspase $\left.3\left(\mathrm{CASP}_{3}\right)^{[25]}\right]$. To isolate RNA, graft particles were immediately snap frozen in liquid nitrogen after extraction and stored at $-80{ }^{\circ} \mathrm{C}$ until processing. Frozen samples were thawed on ice and transferred to a sterile petri dish and cut into very small pieces, approximately the size of a grain of rice. Tissue was transferred to a 5-mL round bottom tube with 1-mL RNeasy Lysis Buffer (RLT) plus (Qiagen) buffer and placed immediately on ice until homogenization with a probe homogenizer (Bio-Gen PRO200). RNA was then extracted with a Qiagen RNeasy MinElute kit according to manufacturer's instructions. RNA quantity and purity were assessed using a plate-reader (Tecan Infinite M200) at 260-nm/280-nm ratio. complementary DNA was reverse transcribed using 25-ng/ $\mu$ L RNA with Moloney Murine Leukemia Virus Reverse Transcriptase (200 U/ $\mu \mathrm{L})$. qRT-PCR was performed with the following primers (all Thermo Fisher): FABP4 (Mm00445878_m1); GPX1 (Mm00492427_m1), CASP3 (Mm01195085_m1), TNF (Mm00438653_m1), and IL1a (Mm04336046_m1). Relative expression was determined by $\Delta \Delta \mathrm{Ct}$ from GAPDH housekeeping gene.

\section{Statistical analysis}

All data are presented as mean and standard deviation (SD) for all groups. The $t$-test was performed to compare groups at single timepoints or differences between two samples in a group at various timepoints with GraphPad Software Prism (GraphPad Software, Inc. San Diego, CA). Statistically significant differences 


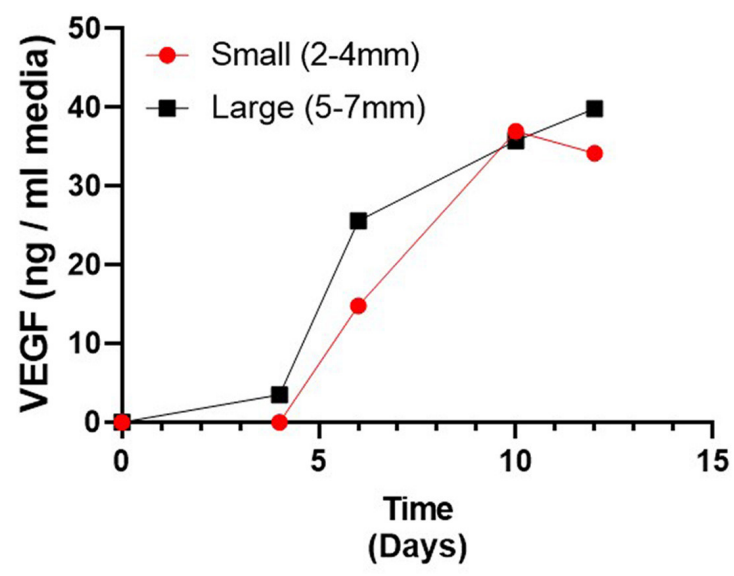

Figure 5. Measurement of VEGF concentration in ex vivo culture media with ELISA. Data are presented as mean $(n=2$ independent biologic replicates per group) with no statistical comparison performed due to low sample size. VEGF: vascular endothelial growth factor ELISA: enzyme-linked immunosorbent assay

were determined using $P$-value less than 0.05. Statistical modeling of intra-animal reproducibility was performed in consultation with the University of Pittsburgh Department of Statistics.

\section{RESULTS}

\section{In vitro analysis of human adipose particle size and hypoxia}

Liposuction cannulas and harvested fat particles analysis

Semi-automated measurement of particle size was verified using glass calibration beads. The results from glass particles demonstrated that the method was consistent, with $8 \%$ and $6.5 \%$ standard deviation for 3.35 - and 2-mm particles, respectively, and the method was able to separate particle diameter differences of $1.35-\mathrm{mm}$ diameter with statistical significance [Figure $4 \mathrm{~A}]$.

The radius of adipose particles harvested with the Bucket, Khouri, Mercedes Tip, and Shippert cannulas were $3.03 \pm 0.78,1.98 \pm 0.92,2.31 \pm 0.85$, and $1.9 \pm 0.59 \mathrm{~mm}$, respectively [Figure $4 \mathrm{~B}$ ]. The range of fat particles diameters was approximately $2-7 \mathrm{~mm}$ [Figure 2]; therefore, the range was divided into two groups for future studies, with small $(2-4 \mathrm{~mm})$ and large $(5-7 \mathrm{~mm})$ sized particles.

\section{VEGF expression in ex vivo model}

An ex vivo study was conducted with fat particles of variable diameter to evaluate the impact of fat particle size on oxygen diffusion limitation and tissue hypoxia. Adipose tissue obtained during panniculectomy was minced with surgical scissors into small and large particle sizes and cultured in equal total amounts in cell culture media. VEGF is an important growth factor for particle revascularization and is expressed by adipose due to hypoxia. Quantification of VEGF concentration in media with ELISA showed increased expression in both particle groups every consecutive day for seven days of culture [Figure 5]. VEGF media concentration was significantly higher in 5-7-mm group compared to the 2-4-mm group at Days 4 and 6 (5928.73 \pm $2572.74 \mathrm{pg} / \mathrm{mL}$ vs. $1507.18 \pm 313.16 \mathrm{pg} / \mathrm{mL}$ and $23,950.61 \pm 2946.86 \mathrm{pg} / \mathrm{mL} v s .15,126.37 \pm 3846.77 \mathrm{pg} / \mathrm{mL}$, respectively, $P<0.01$ ), suggesting that larger particles experience increased hypoxia compared to smaller particles.

\section{In vivo analysis of fat particle survival in mouse autograft model}

Fat particles preparation and transplantation

All experimental animals receiving fat grafts survived well without complications and all surgical incision wounds healed normally. At each timepoint, grafted particles were easily discernable in the subcutaneous 

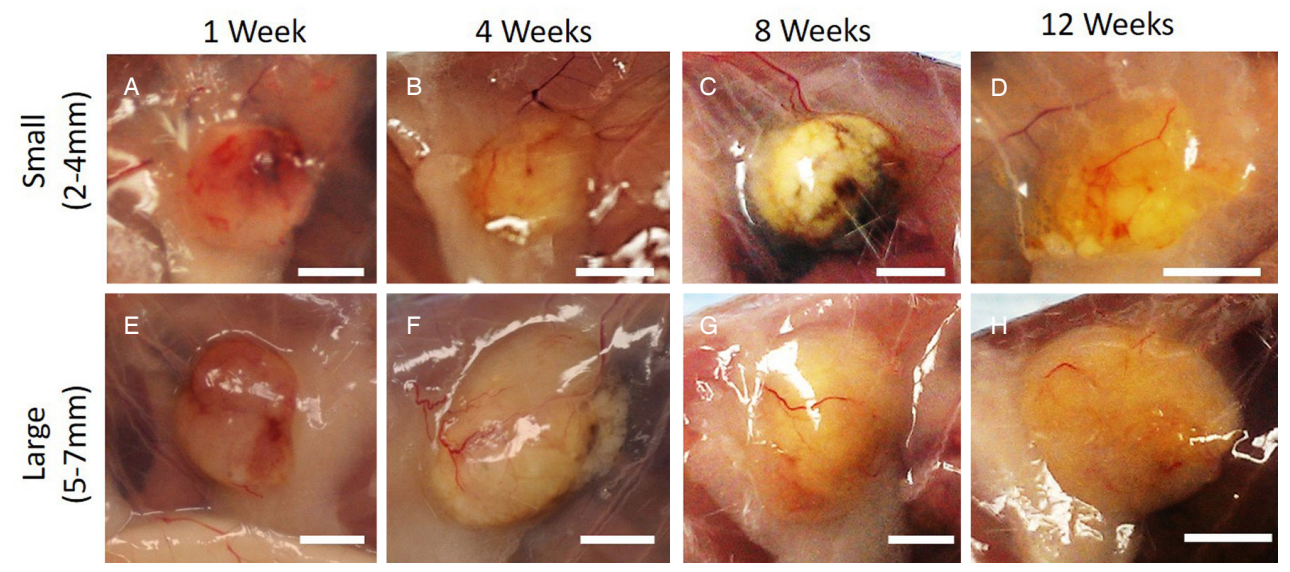

Figure 6. Macroscopic views of grafted fat par ticles of 2-4 mm (A-D) and 5-7 mm (E-H) group at 1, 4, 8, and 12 weeks, respectively, after surgery

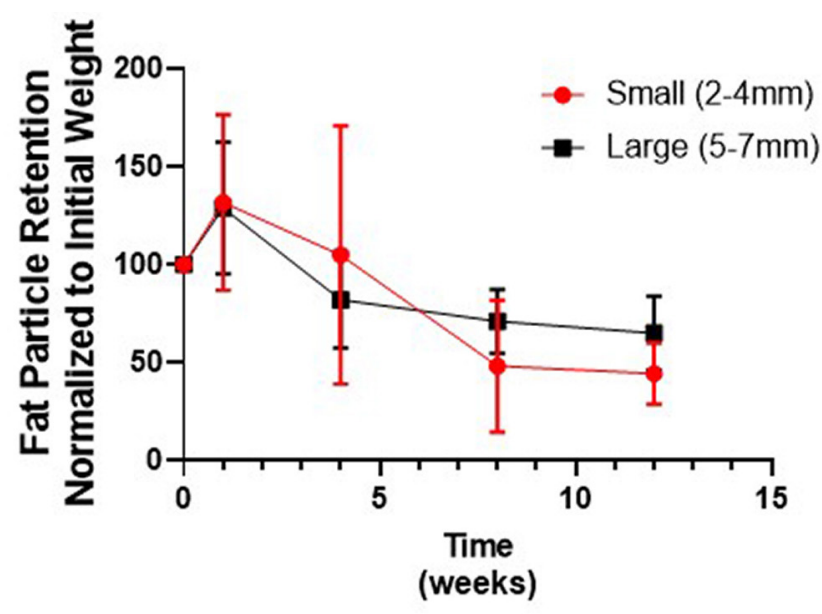

Figure 7. Retention of fat particles normalized to starting weight. Data are presented as the mean percentage of weight retention $\pm \mathrm{SD}$, with 10 replicates per group, pooled from two complete experimental replicates
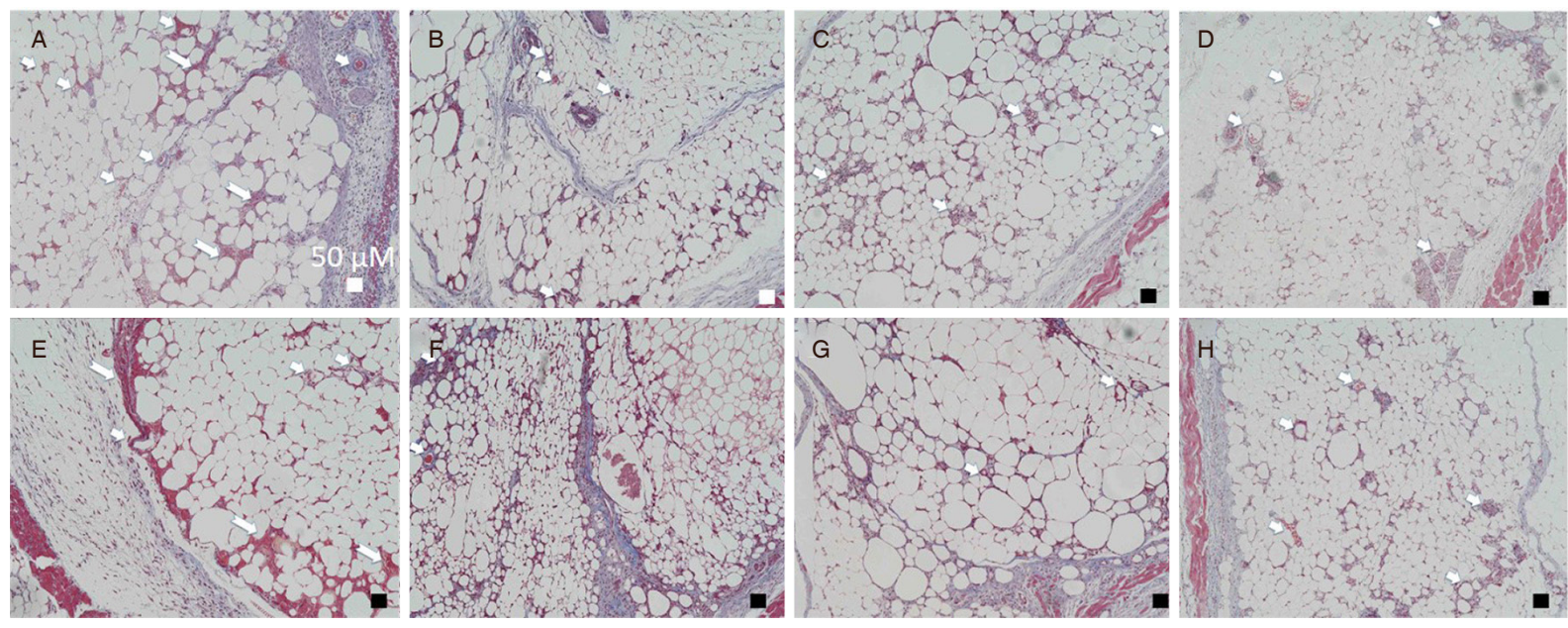

Figure 8. Masson's trichrome staining of explanted fat particles. Fat particles of 2-4 mm (A-D) and 5-7 mm (E-H) groups at 1, 4, 8, and 12 weeks, respectively, after surgery. Small arrows, vessels; Long-tailed arrows, fibrosis. Scale bar, $50 \mu \mathrm{m}$ 


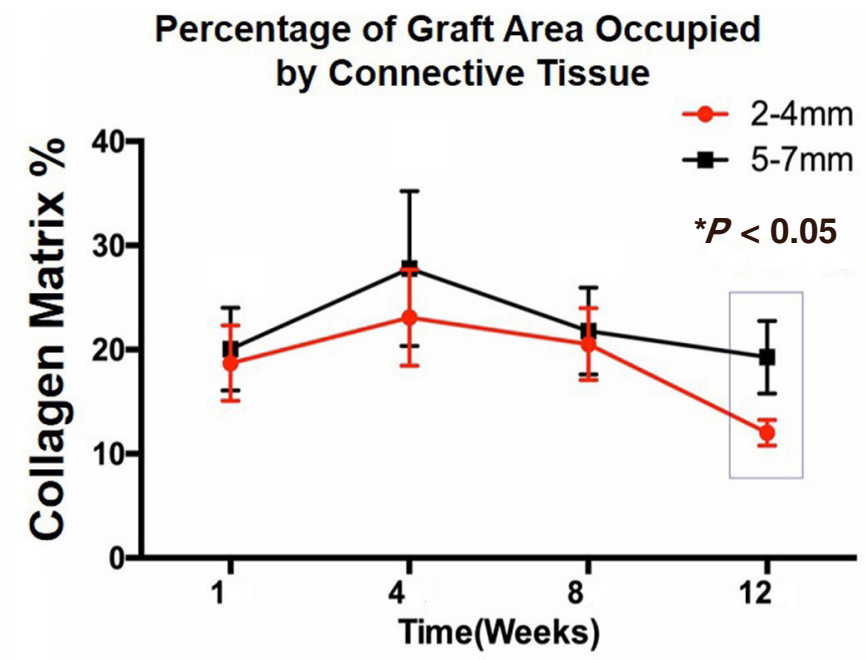

Figure 9. Analysis of fat particle cross-sectional area comprised of connective tissue at each experimental timepoint. Analysis performed by measuring blue collagen as a percentage of total particle area. ${ }^{\star} P<0.05$ at 12 weeks

space and were encapsulated in thin membranes of fibrous septae [Figure 6A-H]. At one week, fat parcels in both small and large particle groups demonstrated bleeding, potentially indicating more permeable blood vessels and ongoing inflammation ${ }^{[26]}$. At later timepoints, angiogenesis and blood vessel stabilization were observed in both particle groups. Considering the small size of fat particles, retention was calculated in terms of weight, as we could measure this with an analytic balance with greater sensitivity than available methods to measure volume. For each sample, the percent retention was determined by normalizing the explantation weight to the initial weight of each particle recorded before grafting. There was no significant difference in particle retention at any timepoint for 2-4- and 5-7-mm particles [Figure 7].

\section{Histologic assessment of grafted fat particle in mice}

Masson's trichrome staining revealed morphological changes of connective tissue components including extracellular matrix and fibrosis over time with interesting differences in adipocyte sizes between small and large particles [Figure 8A-H]. In general, adipocyte necrosis was observed by four weeks, as evident by large spaces of oil, with increased presence at eight weeks and near complete resorption by 12 weeks. In contrast, small and irregularly shaped adipocytes were evident at four weeks and increased in frequency at 8 weeks. The ratio of connective tissue (collagen indicated with blue) to whole area was calculated for each particle group [Figure 9]. Small particles had increased density of collagens at four and eight weeks (23\% \pm $4 \%$ and $20 \% \pm 3 \%)$ but decreased density at 12 weeks $(12 \% \pm 1 \%, P<0.05)$ as it was replaced by adipocytes. In contrast, particles of 5-7 mm had significantly increased collagen matrix at 12 weeks compared to small particles $(P<0.05)$, which may indicate that additional replacement by adipocytes would occur at a later timepoint.

\section{Gene expression analysis}

There were no statistical differences between measured gene expression between small and large particles for any gene at any timepoint, perhaps due to high inter-sample variability in each group [Figure 10]. In general, both particles had low FABP4 expression at one week, suggesting low adipocyte function, and increased expression levels at each consecutive timepoint, suggesting adipocyte regeneration within the grafts [Figure 10A]. Glutathione peroxidase 1 is an enzyme which catalyzes the reduction of organic hydroperoxides and hydrogen peroxide $\left(\mathrm{H}_{2} \mathrm{O}_{2}\right)$ and thereby protects cells against oxidative damage. GPX1 is therefore upregulated by cells as a defense mechanism against reactive oxygen species. Small-sized particles had increased GPX1 expression at one week [Figure 10B] and simultaneously decreased expression of 
A

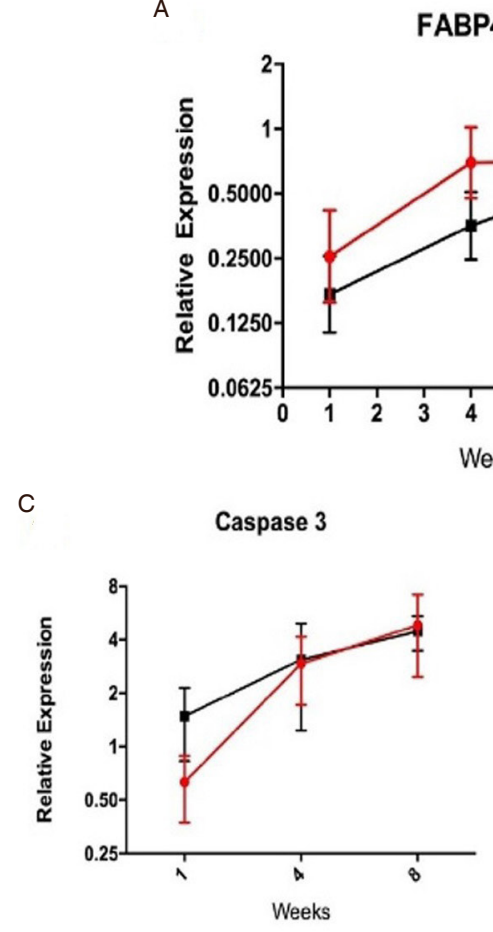

B

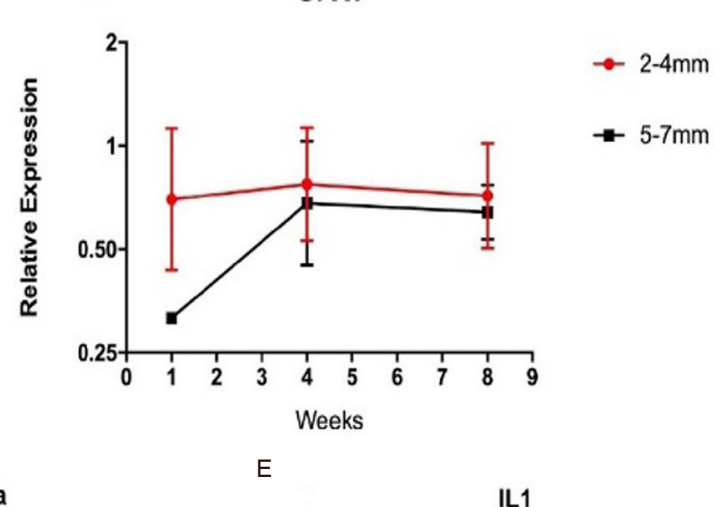

Figure 10. Relative expression of adipocyte function (FABP4), protection from oxidative stress (GPX1), apoptosis (Caspase 3), and inflammation (TNF $\alpha$ and IL1) at one, four, and eight weeks post-grafted syngeneic fat particles. FABP4: fatty acid binding protein 4; GPX1: glutathione peroxidase; TNF $\alpha$ : tumor necrosis factor; IL1: interleukin 1

Caspase 3, which indicates active apoptotic pathways. Together, these results suggest reduced tissue survival in the larger particle group at early timepoints but equivalent and continuous tissue turnover throughout the regenerative process [Figure $10 \mathrm{C}$ ]. We further speculate continuous infiltration of immune cells and tissue remodeling throughout fat graft regeneration due to consistently high levels of inflammatory genes that are typical of macrophages [Figure $10 \mathrm{D}$ and E]. Graft tissue replacement by circulating cells has been documented in other studies using fluorescently labeled bone-marrow cells; thus, these observations are in line with current observations in the literature ${ }^{[27,28]}$.

\section{DISCUSSION}

Fat is a highly variable tissue that contains soft lipid-filled lobules and tough, fibrous connective tissue. Fat varies from patient to patient and across anatomical regions within a patient. To extract fat, a blunt end, hollow surgical cannula is passed many times through the tissue and, when suction is applied, small fat parcels are drawn into the cannula. Fat particles are defined as intact globules of adipocytes, microvasculature, and connective tissues. Particles do not occur in native adipose tissue, but rather are cut and formed during the harvesting step of fat grafting. Various cannula are commercially available with fixed aperture size and configuration to meet variable fat tissue needs. To obtain tissue for secondary grafting procedures, surgeons may attempt to use the smallest cannula possible, with the hypothesis that small fat particles will produce superior fat grafting results. However, our results suggest that the extra labor required to use small aperture cannula for the purposes of reducing particle size may not be necessary (or even helpful).

A common problem with current lipoplasty cannula is clogging due to parcels of fat becoming lodged in cannula apertures requiring surgeons to test multiple cannulas mid-surgery to find one that is appropriate. This cannula exchange process is tedious, time consuming, and requires additional operating room staff for 
assistance. The primary driver of fat particle size manipulation is to avoid "large" fat particles in subsequent grafting procedures. These particles may clog injection cannula or have insufficient oxygen and nutrient diffusion to the interior particle core, resulting in large necrosis and oil cyst formation. These oil cysts are eventually resorbed through macrophage clearance resulting in graft tissue loss and inferior long-term fat grafting outcomes.

Clinically, it has been difficult to parse out the impact of fat particle size on graft retention from other confounding variables such as trauma associated with harvest or injection. Our study aimed to isolate the variable of fat particle size to determine the impact of fat particle size on in vivo survival and viability of fat grafts. To do this, an immunocompetent, inbred mouse model was selected to avoid donor variability with human adipose tissue. Adipose particles for grafting were prepared from whole subcutaneous fat pads by mincing tissue with surgical scissors. This allowed for consistent particle samples of known diameter while eliminating possible trauma from harvest cannulas. Study outcomes included graft retention, tissue histology, and measured genetic markers of inflammation, apoptosis, and tissue regeneration (qPCR).

Our data suggest that fat particles below $7 \mathrm{~mm}$ produce similar fat grafting outcomes despite increased hypoxia in 5-7-mm particles compared to 2-4-mm diameter particles. The histological findings from our study show that the small fat particles group led to milder necrosis and more neovascularization (especially in the early stages) while larger particles experienced increased necrosis and areas with irregular adipocyte morphology. Interestingly, while small fat particles showed superior architecture at the one-week study timepoint, this was reversed at the 12 -week timepoint, with large fat particle grafts containing larger adipocytes and a better-organized histological structure. This may suggest that early adipocyte necrosis leading to extracellular matrix deposition through fibrosis results in a scaffold for tissue regeneration from circulating progenitor cells, as was also proposed by Del Vecchio et el. ${ }^{[29]}$, who found that smaller particles may lack connective tissue for structural support of adipocytes and proliferating stem cells. The regenerative role of fascia, connective tissue, and extracellular matrix components of the fat graft is often overlooked and yet to be fully understood. This study, along with our previous published work, aims to fill this knowledge gap.

Cumulatively, our studies suggest that, independent of adipose tissue quality, regenerative adipogenesis is highly dependent on the tissue scaffold, followed by angiogenesis, and lastly adipose induction or adipogenesis with all components playing important sequential and dynamic roles. It is hypothesized that finding the balance of these components will depend largely on the recipient site and the amount and nature of the host tissue in that region. As previous studies have demonstrated the angiogenic nature of proteins present in the fascia/connective tissue which angiogenesis occurs prior to adipogenesis, future studies on particle size could also include immunohistochemistry for CD31 to see if the larger particle group goes through this phase of angiogenesis at a different rate from small particles.

The results from this study have been corroborated elsewhere where particle size has shown no significant impact on long-term outcomes when the particle diameter is less than or equal to $7 \mathrm{~mm}^{[11,12]}$. Fisher et al. ${ }^{[30]}$ transplanted lipoaspirate filtered with 500 and $800 \mu \mathrm{m}$ filters, whose average sizes were approximately 5.9 and $3.2 \mathrm{~mm}$, and found there was no statistically significant difference between each other. Ozsoy et al. ${ }^{[31]}$ compared lipoaspirate from human pannus harvested with 2-, 3-, and 4-mm diameter cannulas, and showed that the 4-mm cannulas had the highest level of adipocyte viability. Erdim et al. ${ }^{[15]}$ evaluated viability of fat grafts, which was harvested from the abdomen of 10 consecutive patients using 2-, 4-, and 6-mm cannulas, by using colleganase digestion, supravital staining, and adipocyte counting using a haemocytometer. The results demonstrate a higher number of viable adipocytes from the aspirate occurred with the 6-mm cannula as opposed to the 2 - and 4-mm cannulas. Therefore, the impact of harvesting cannula diameter may not be a critical factor on ultimate graft retention when fat particles are $\leq 7 \mathrm{~mm}$ in diameter, which we found to be the case in four commonly used lipoplasty harvesting cannula. 
In conclusion, Fat particles with size below $7 \mathrm{~mm}$ in diameter do not alter graft survival in an immunocompetent mouse model. While larger fat particles experience early hypoxia and adipocyte loss, the remaining tissue serves as a scaffold for regeneration by circulating cells. This study provides important evidence on the fat grafting process, suggesting greater flexibility in lipoplasty cannula selection for fat grafting procedures than previously thought, allowing increased harvest yields and decreased effort and time.

\section{DECLARATIONS}

\section{Authors' contributions}

Performed partial examinations, analyzed and interpreted the research data: Yang X

Assisted with manuscript preparation: Egro FM, Nerone WV, Yousefpour M

Assisted with animal experiments, performed qRT-PCR experiments and assisted with data analysis: Jones T Assisted with submission of the manuscript: Yousefpour M

Participated in overall study design and assisted with acquisition of human tissue specimens: Gusenoff JA, Rubin JP

Corresponding author is responsible for ensuring that all listed authors have approved the manuscript before submission and that all authors receive the submission and all substantive correspondence with editors: Kokai LE

All authors read and approved the final manuscript.

\section{Availability of data and materials}

The datasets used and/or analyzed during the current study are available from the corresponding author on reasonable request.

\section{Financial support and sponsorship}

This work was funded by a Pilot Funding Program for Early Stage Medical Technology Research and Development grant from the Center for Medical Innovation, Swanson School of Engineering, University of Pittsburgh (award no. F_062-2013) and by National Institutes of Health for Dr. Rubin, Department of Plastic Surgery; University of Pittsburgh School of Medicine (award no. 5R01CA114246).

\section{Conflicts of interest}

All authors declare that they are bound by confidentiality agreements that prevent them from disclosing their conflicts of interest in this work.

\section{Ethical approval and consent to participate}

All animal experiments were performed under approved protocols by the University of Pittsburgh Institutional Animal Care and Use Committee (Protocol\# 12080782).

\section{Consent for publication}

Not applicable

\section{Copyright}

(c) The Author(s) 2020.

\section{REFERENCES}

1. Cosmetic surgery national data bank statistics. Aesthetic Surg 2018;38:1-24.

2. Coleman SR. Structural fat grafting: more than a permanent filler. Plast Reconstr Surg 2006;118:108S-20S.

3. Gause TM, Kling RE, Sivak WN, Marra KG, Rubin JP, et al. Particle size in fat graft retention: a review on the impact of harvesting technique in lipofilling surgical outcomes. Adipocyte 2014;3:273-9.

4. Gir P, Brown SA, Oni G, Kashefi N, Mojallal A, et al. Fat grafting: evidence-based review on autologous fat harvesting, processing, reinjection, and storage. Plast Reconstr Surg 2012;130:249-58. 
5. Smith P, Adams WP Jr, Lipschitz AH, Chau B, Sorokin E, et al. Autologous human fat grafting: effect of harvesting and preparation techniques on adipocyte graft survival. Plast Reconstr Surg 2006;117:1836-44.

6. Sommer B, Sattler G. Current concepts of fat graft survival: histology of aspirated adipose tissue and review of the literature. Dermatol Surg 2000;26:1159-66.

7. Crawford JL, Hubbard BA, Colbert SH, Puckett CL. Fine tuning lipoaspirate viability for fat grafting. Plast Reconstr Surg 2010;126:1342-8.

8. Rohrich RJ, Sorokin ES, Brown SA. In search of improved fat transfer viability: a quantitative analysis of the role of centrifugation and harvest site. Plast Reconstr Surg 2004;113:391-5.

9. Cheriyan T, Kao HK, Qiao X, Guo L. Low harvest pressure enhances autologous fat graft viability. Plast Reconstr Surg 2014;133:1365-8.

10. Yamaguchi M, Matsumoto F, Bujo H, Shibasaki M, Takahashi K, et al. Revascularization determines volume retention and gene expression by fat grafts in mice. Exp Biol Med (Maywood) 2005;230:742-8.

11. Carpaneda CA, Ribeiro MT. Percentage of graft viability versus injected volume in adipose autotransplants. Aesthetic Plast Surg 1994; $18: 17-9$.

12. Khouri RK Jr, Khouri RE, Lujan-Hernandez JR, Khouri KR, Lancerotto L, et al. Diffusion and perfusion: the keys to fat grafting. Plast Reconstr Surg Glob Open 2014;2:e220.

13. Asken S. Autologous fat transplantation: micro and macro techniques. Am J Cosmetic Surg 1987;4:111-21.

14. Agris J. Autologous fat transplantation: a 3-year study. Am J Cosmetic Surg 1987;4:95-102.

15. Erdim M, Tezel E, Numanoglu A, Sav A. The effects of the size of liposuction cannula on adipocyte survival and the optimum temperature for fat graft storage: an experimental study. J Plast Reconstr Aesthet Surg 2009;62:1210-4.

16. Kirkham JC, Lee JH, Medina MA, McCormack MC, Randolph MA, et al. The impact of liposuction cannula size on adipocyte viability. Ann Plast Surg 2012;69:479-81.

17. Fagrell D, Eneström S, Berggren A, Kniola B. Fat cylinder transplantation: an experimental comparative study of three different kinds of fat transplants. Plast Reconstr Surg 1996;98:90-6.

18. Wree A, Mayer A, Westphal S, Beilfuss A, Canbay A, et al. Adipokine expression in brown and white adipocytes in response to hypoxia. J Endocrinol Invest 2012;35:522-7.

19. Van Pham P, Vu NB, Phan NK. Hypoxia promotes adipose-derived stem cell proliferation via VEGF. Biomed Res Ther 2016;3:1-7.

20. Wojciechowicz K, Gledhill K, Ambler CA, Manning CB, Jahoda CA. Development of the mouse dermal adipose layer occurs independently of subcutaneous adipose tissue and is marked by restricted early expression of FABP4. PLoS One 2013;8:e59811.

21. Hotamisligil GS. Inflammation and metabolic disorders. Nature 2006;444:860-7.

22. Mahmood DFD, Abderrazak A, El Hadri K, Simmet T, Rouis M. The thioredoxin system as a therapeutic target in human health and disease. Antioxid Redox Signal 2013;19:1266-303.

23. Lu J, Holmgren A. Thioredoxin system in cell death progression. Antioxid Redox Signal 2012;17:1738-47.

24. Linares M, Marín-García P, Martínez-Chacón G, Pérez-Benavente S, Puyet A, et al. Glutathione peroxidase contributes with heme oxygenase-1 to redox balance in mouse brain during the course of cerebral malaria. Biochim Biophys Acta 2013;1832:2009-18.

25. Wu Y, Wang D, Wang X, Wang Y, Ren F, et al. Caspase 3 is activated through caspase 8 instead of caspase 9 during H2O2-induced apoptosis in heLa cells. Cell Physiol Biochem 2011;27:539-46.

26. Park SA, Jeong S, Choe YH, Hyun YM. Sensing of vascular permeability in inflamed vessel of live animal. J Anal Methods Chem 2018;2018:5797152.

27. Zhao J, Yi C, Li L, Zheng Y, Wu K, et al. Observations on the survival and neovascularization of fat grafts interchanged between C57BL/6-gfp and C57BL/6 mice. Plast Reconstr Surg 2012;130:398e-406e.

28. Doi K, Ogata F, Eto H, Kato H, Kuno S, et al. Differential contributions of graft-derived and host-derived cells in tissue regeneration/ remodeling after fat grafting. Plast Reconstr Surg 2015;135:1607-17.

29. Del Vecchio D, Rohrich RJ. A classification of clinical fat grafting: different problems, different solutions. Plast Reconstr Surg 2012;130:511-22.

30. Fisher C, Grahovac TL, Schafer ME, Shippert RD, Marra KG, et al. Comparison of harvest and processing techniques for fat grafting and adipose stem cell isolation. Plast Reconstr Surg 2013;132:351-61.

31. Ozsoy Z, Kul Z, Bilir A. The role of cannula diameter in improved adipocyte viability: a quantitative analysis. Aesthet Surg J 2006;26:287-9. 